University of Nebraska - Lincoln

DigitalCommons@University of Nebraska - Lincoln

July 2001

\title{
The Blazhko Effect of the RR Lyrae Star FM Persei
}

Kevin M. Lee

University of Nebraska-Lincoln, klee6@unl.edu

Edward G. Schmidt

University of Nebraska-Lincoln, eschmidt1@unl.edu

Follow this and additional works at: https://digitalcommons.unl.edu/physicsschmidt

Part of the Physics Commons

Lee, Kevin M. and Schmidt, Edward G., "The Blazhko Effect of the RR Lyrae Star FM Persei" (2001). Edward Schmidt Publications. 6.

https://digitalcommons.unl.edu/physicsschmidt/6

This Article is brought to you for free and open access by the Research Papers in Physics and Astronomy at DigitalCommons@University of Nebraska - Lincoln. It has been accepted for inclusion in Edward Schmidt Publications by an authorized administrator of DigitalCommons@University of Nebraska - Lincoln. 
Publications of the Astronomical Society of the Pacific, 113:835-838, 2001 July

(C) 2001. The Astronomical Society of the Pacific. All rights reserved. Printed in U.S.A.

\title{
The Blazhko Effect of the RR Lyrae Star FM Persei
}

\author{
Kevin M. Lee and Edward G. Schmidt \\ Behlen Observatory, Department of Physics and Astronomy, University of Nebraska, Lincoln, NE 68588-0111; klee6@unl.edu \\ Received 2000 June 11; accepted 2001 April 3
}

\begin{abstract}
We have obtained 615 new $V$ and $R$ observations of the RR Lyrae star FM Per. A period search identified a primary period of 0.489256 days; however, the light curve of FM Per cannot be well described by a single period. We conclude that FM Per demonstrates the Blazhko effect with a Blazhko period of approximately 122 days.
\end{abstract}

\section{INTRODUCTION}

We have undertaken a survey of 107 Bailey type ab RR Lyrae stars to determine the frequency and characteristics of multiperiodic behavior among such objects. Multiperiodicity will be identified by observing the stars near the phase of maximum light and selecting those in which the magnitude or phase of maximum does not repeat well from one cycle to another. As of now, observations have been finished for about two-thirds of the survey sample. Rather than await the conclusion of the survey, results for interesting individual stars from the sample will be published as they are completed. This is the second in a series of such papers, the first being on V442 Her (Schmidt \& Lee 2000).

Most RR Lyrae stars repeat their light curves with remarkable regularity. However, roughly $30 \%$ of known RR Lyrae stars display light curves which change form on relatively short timescales because of multiperiodicity; see the review by Szeidl (1988). The vast majority of these multiperiodic RR Lyrae stars exhibit what is known as the Blazhko effect (Blazhko 1907). The Blazhko effect is the periodic modulation of either the amplitude alone or the amplitude and phase of maximum light. Stars which demonstrate variations of the amplitude alone often have very little light-curve variation at phases far from maximum. Smith (1995, Table 5.2) lists 46 stars known to exhibit the Blazhko effect. The Blazhko periods in this table range from 10.9 to 533 days, but most have periods between 20 and 200 days. There is no commonly accepted theory that explains the Blazhko effect.

RR Lyrae stars may also have irregular light curves as a result of double-mode pulsation. These stars are known as RRd stars (in the terminology of Nemec 1985). The mechanism for this variation (stars pulsating in the fundamental mode and the first-overtone mode simultaneously) is well understood. Typically, the period ratio for these two processes, $P_{1} / P_{0}$, is around 0.743-0.748 (Cox 1987). Thus, light-curve variations due to double-mode pulsation manifest themselves on a shorter timescale than those due to the Blazhko effect, and it is easy to discriminate between the two. One would also expect lightcurve scatter from double-mode pulsation to be found not just near maximum light but at all phases. At present, at least five RRd field stars have been identified (Jerzykiewicz \& Wenzel 1977; Clement et al. 1991; Garcia-Melendo \& Clement 1997; Moskalik 2000).

FM Per (GSC 33311127) is located at $\alpha_{2000}=4^{\mathrm{h}} 03^{\mathrm{m}} 27^{\mathrm{s}}$, $\delta_{2000}=47^{\circ} 59^{\prime} 52^{\prime \prime}$. Very little information exists on FM Per in the literature. It is listed in the General Catalogue of Variable Stars (GCVS; Kholopov 1985, 1987) as an RRab star with a period of 0.4892014 days. The comments section of the GCVS notes that its period possibly varies. A search of the SIMBAD database revealed that the only recently published observations were 19 photometric observations made by one of the authors (Schmidt et al. 1995). Schmidt confirmed the star as a type RRab and derived the period of $0.4896 \pm 0.0002$ days. No explanation was offered for the discrepancy between this value and that of the GCVS. However, Schmidt does note that the Blazhko effect can cause periods to be in error by more than their estimated error.

\section{OBSERVATIONS}

We observed FM Per 437 times on 30 nights between 1996 September 17 (JD 2,450,343) and 1997 March 31 (JD 2,450,538), an interval of 195 days. During a second observing season, we obtained 178 points between 1997 August 9 (JD 2,450,669) and 1998 January 1 (JD 2,450,814), an interval of 145 days. The observing system consisted of a Photometrics, Ltd., TI4849, $390 \times 584$ pixel, cryogenically cooled CCD detector mounted at the Cassegrain focus of the Behlen Observatory $76 \mathrm{~cm}(\mathrm{f} / 13.5)$ telescope. The field of view is $2.9 \times 4 ! 3$, with a scale of 0.44 pixel $^{-1}$. Observations were made through $V$ and $R$ filters and transformed to the system of Cousins (1976). Because of the large number of points involved, the original photometric data will not be listed here. It is available in the IAU Archives of Unpublished Observations of Variable Stars in archive 330E. See Breger, Jaschek, 
TABLE 1

Coordinates and Magnitudes of the Program Stars

\begin{tabular}{cccccc}
\hline \hline Star & GSC No. & $\alpha_{2000}$ & $\delta_{2000}$ & $V$ & $R$ \\
\hline $\mathrm{C} 1 \ldots \ldots$ & 33311465 & 4320.6 & 480009 & 13.604 & 13.030 \\
$\mathrm{C} 2 \ldots \ldots$ & 33311245 & 4314.7 & 480043 & 12.859 & 12.386 \\
$\mathrm{C} 3 \ldots \ldots$ & 33311671 & 4313.2 & 480054 & 12.348 & 11.773 \\
\hline
\end{tabular}

Note. - Units of right ascension are hours, minutes, and seconds, and units of declination are degrees, arcminutes, and arcseconds.

\& Dubois (1990) for a description of the archives and how to access them.

The Behlen Observatory CCD photometry system and observational techniques were the same as described earlier (Schmidt 1991 and references therein). However, it should be emphasized that differential magnitudes were calculated for FM Per by using other stars on the same CCD frames as comparison stars. Thus accurate differential magnitudes were obtained even under inferior photometric conditions. Color terms for the transformation to the standard system were then determined by observing standard stars from Landolt (1983); average values of these color terms over intervals of several months were used to correct all nights within the interval. The quality of the photometry may be judged by the rms scatter between the comparison stars, which was 0.017 in $V$ and 0.016 in $R$. Table 1 lists the coordinates and magnitudes on the standard system of the three comparison stars.

\section{DATA ANALYSIS}

A period search was performed on the data using phase dispersion minimization (PDM) analysis (Stellingwerf 1978). In this method the data are binned according to a series of trial periods and a statistic $\theta$ is calculated for each trial period. The statistic $\theta$ is defined as the weighted average of all individual bin variances divided by the variance of the entire data set.

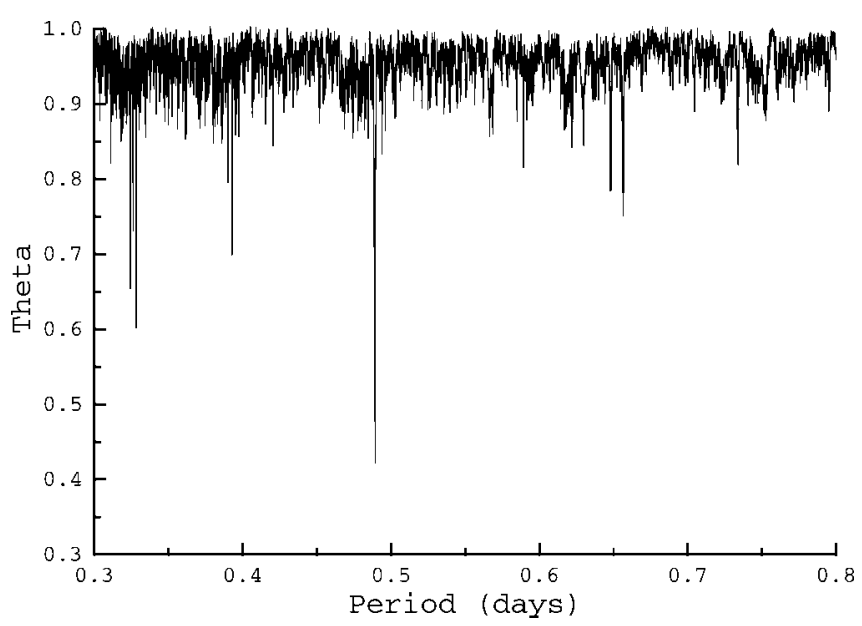

Fig. 1.-Power spectrum obtained from PDM analysis of the FM Per $V$ data set.
The data were grouped into phase bins of $N_{b}=5, N_{c}=2$ in Stellingwerf's nomenclature. The graph of $\Theta$ versus trial period obtained from this routine on the $V$ data set is shown in Figure 1 , where a periodic signal is identified by a significant minimum in $\Theta$. The most significant minimum corresponds to a period of 0.489256 days. The $R$ data set yielded an identical result.

The light curve obtained from folding our data with the period of 0.489256 days is shown in Figure 2. Visual inspection of this light curve is sufficient to convince one that FM Per is multiperiodic and that the secondary periodicity has a fairly large amplitude. Although the magnitude of maximum light varies considerably, the phase of maximum light does not. The phase of maximum light drifted toward larger phases for more recently taken data when the GCVS period was used to form the light curve. The mean intensity magnitudes of the light curves were determined to be $\langle V\rangle=12.777$ and $\langle R\rangle=12.163$.

A sequential graphing of the light-curve data points in chronological order allowed one to easily view a slow cyclic variation in the magnitude of maximum light. Thus we next investigated this variation to determine the secondary periodicity.Table 2 lists the Julian Dates and magnitudes of FM Per at maximum light obtained from the $V$ photometric data. Two different types of maximum light are identified: those for which the observations are sufficient in number and spacing so that the maxima is well defined (WD) and those for which the maxima may only be estimated (E). If not well defined, the magnitude of maximum light was estimated on a given night if there was a data point within 0.02 phase units of the phase of maxima. The magnitude of the point within 0.02 phase units was adopted as the maximum value. From the data inTable 2, one can determine that the amplitude of the Blazhko effect at maximum light is at least $0.42 \mathrm{mag}$ in $V$, and we determined a lower limit for the $R$ amplitude of 0.34 mag.

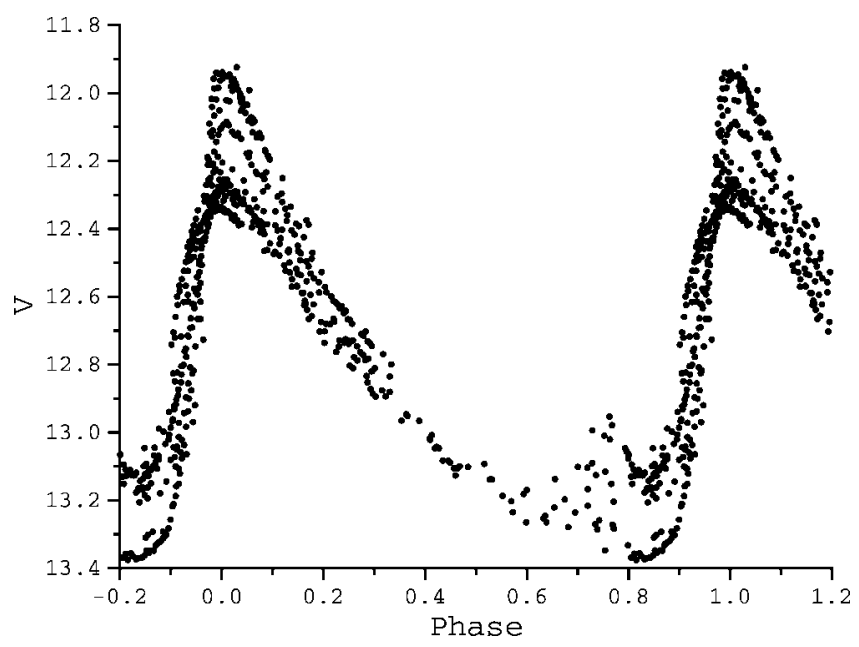

FIG. 2.-Light curve formed from the FM Per $V$ data set where the phases have been determined by $\phi=(\mathrm{JD}-2,450,389.896) / 0.489256$. 
TABLE2

Magnitudes of FM Per at Maximum Light

\begin{tabular}{|c|c|c|c|c|c|}
\hline $\begin{array}{l}\text { Heliocentric Julian Date } \\
\qquad(2,440,000+)\end{array}$ & $\begin{array}{c}V \text { Magnitude } \\
\text { (of Maximum Light) }\end{array}$ & $\begin{array}{l}\text { Definition of Maximum } \\
\text { (WD or E) }\end{array}$ & $\begin{array}{l}\text { Heliocentric Julian Date } \\
\qquad(2,440,000+)\end{array}$ & $\begin{array}{c}V \text { Magnitude } \\
\text { (of Maximum Light) }\end{array}$ & $\begin{array}{l}\text { Definition of Maximum } \\
\text { (WD or E) }\end{array}$ \\
\hline $10,364.390$ & 12.114 & $\mathrm{E}$ & $10,494.593$ & 12.225 & $\mathrm{E}$ \\
\hline $10,389.896 \quad \ldots \ldots \ldots \ldots$ & 12.286 & WD & $10,496.569 \ldots$ & 12.289 & $\mathrm{E}$ \\
\hline $10,390.875 \quad \ldots \ldots \ldots \ldots$ & 12.277 & WD & $10,514.651 \ldots$ & 12.341 & WD \\
\hline $10,412.895 \quad \ldots \ldots \ldots \ldots$ & 12.255 & $\mathrm{E}$ & $10,515.631 \quad \ldots \ldots \ldots \ldots$ & 12.334 & WD \\
\hline $10,413.876 \quad \ldots \ldots \ldots \ldots$ & 12.264 & WD & $10,538.637 \ldots \ldots$ & 12.255 & WD \\
\hline $10,415.831 \quad \ldots \ldots \ldots \ldots$ & 12.282 & WD & $10,709.886$ & 11.924 & WD \\
\hline $10,426.600 \ldots \ldots$ & 12.176 & $\mathrm{E}$ & $10,763.695$ & 12.294 & $\mathrm{E}$ \\
\hline $10,437.850 \quad \ldots \ldots \ldots \ldots$ & 12.085 & WD & $10,777.889$ & 12.312 & WD \\
\hline $10,447.639 \quad \ldots \ldots \ldots \ldots$ & 11.980 & WD & $10,778.860$ & 12.283 & WD \\
\hline $10,465.730 \quad \ldots \ldots \ldots \ldots$ & 11.958 & $\mathrm{E}$ & $10,809.690 \quad \ldots \ldots \ldots \ldots$ & 11.994 & WD \\
\hline $10,466.706 \quad \ldots \ldots \ldots \ldots$ & 11.940 & WD & $10,814.580 \quad \ldots \ldots \ldots \ldots$ & 12.024 & WD \\
\hline $10,468.671 \ldots \ldots \ldots \ldots$ & 11.949 & WD & & & \\
\hline
\end{tabular}

An examination of the values inTable 2 strongly suggests that the Blazhko effect, rather than double-mode pulsation, is present. On four occasions maxima were observed on consecutive nights $(10,389-10,390,10,412-10,413$, $10,465-10,466$, and 10,514-10,515). On all four pairs of nights the magnitude of the maximum on the second night was very close to that obtained on the first night of the pair. Although one would not expect this if FM Per were a double-mode pulsator, we nonetheless investigated this possibility. In the case where the 0.4892 day period was the fundamental period and the period ratio $P_{1} / P_{0}$ was about 0.75 , there would be a first overtone at approximately 0.365 days. However, no local minimum corresponding to this value is present in Figure 1. There is a local minimum at $P=0.393$ days which we interpret as $P=2 /\left(2 \nu_{0}+1\right)$. We also considered the possibility that FM Per was a typical double-mode pulsator where the first-overtone mode pulsation $P_{1}$ is dominant and $P_{0}$ would be approximately

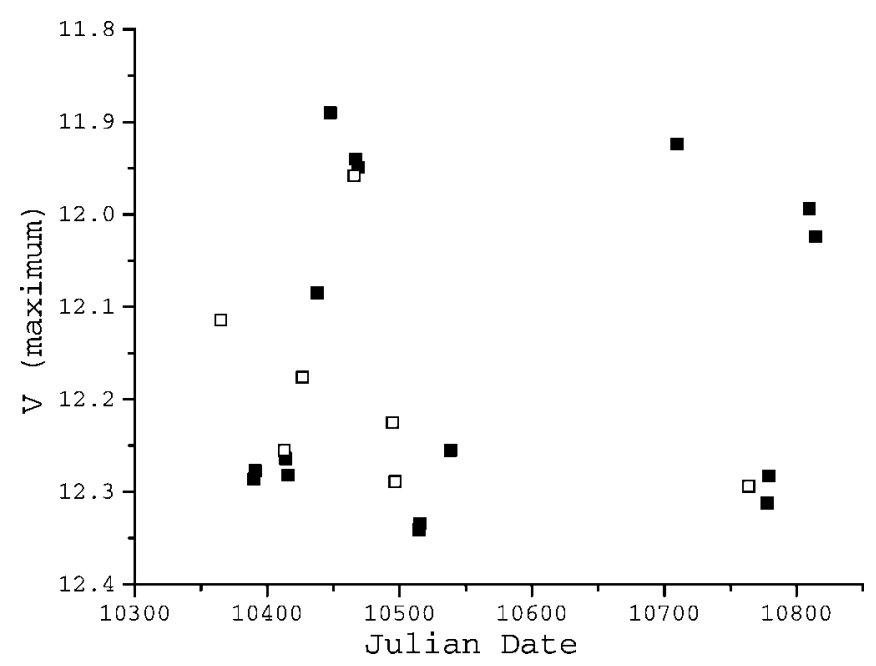

FIG. 3. $-V$ magnitudes of maximum light vs. Julian Date. The solid squares represent well-defined maxima, and the open squares represent estimated maxima.
0.65 days. In this case, on the second night of a consecutive night pair $P_{1}$ has gone through 1 day/0.4892 days $\approx 2.0$ cycles to return to maxima. The period $P_{1}$ would have gone through $1 \mathrm{day} / 0.65$ days $\approx 1.5$ cycles. Although there is a local minimum at $P=0.656$ days in Figure 1, we interpret this as the alias of the fundamental period $P=2 /\left(\nu_{0}+1\right)$.

Figure 3 shows the plot of the magnitudes of maximum light versus Julian Date. One can see from the slow variation in the magnitudes of maximum light that the Blazhko period must be relatively long. We define a "Blazhko curve" as a light curve whose data points are the magnitudes and epochs of maximum light and the phases are determined using the Blazhko period. Thus the Blazhko curve shows the magnitude at maximum light in the primary pulsation cycle plotted as a function of phase in the Blazhko cycle. To identify the Blazhko period, Blazhko curves were formed for a variety of trial Blazhko

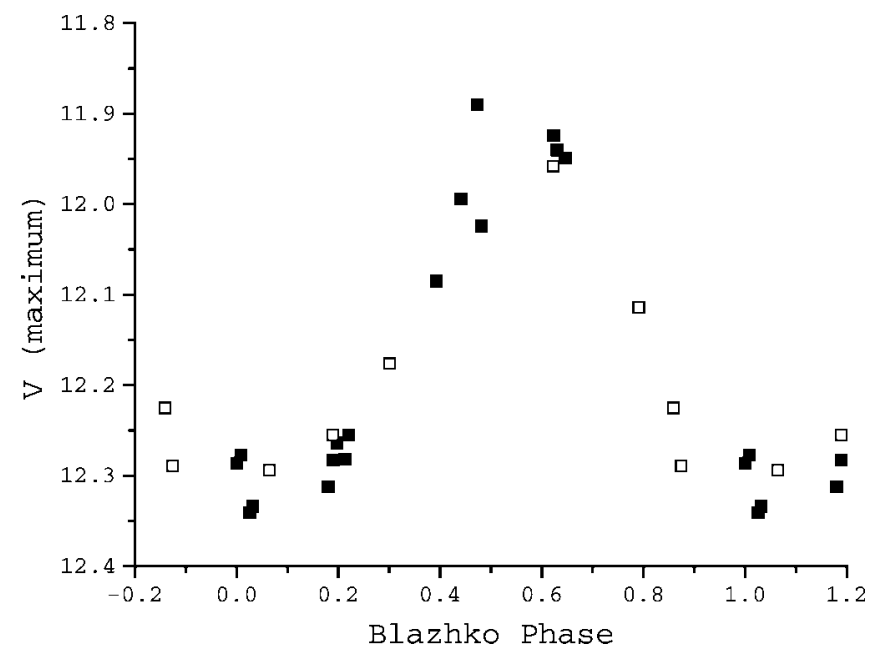

FIG. 4.-Blazhko curve formed from the $V$ magnitudes of maximum light. The Blazhko phases were determined from $\phi=(\mathrm{JD}-2,450,389.896) / 122$. The solid squares represent well-defined maxima, and the open squares represent estimated maxima. 
periods. A Blazhko period of 122 days yielded the best light curve, which is shown in Figure 4. We estimate an uncertainty of \pm 2 days.

We attempted to verify the approximately 122 day Blazhko period using formal period searching techniques. We formed a mean light curve for the data in Figure 2 using least-squares techniques and subtracted the mean light curve from the original data at each phase. We then searched for periodicities in the residuals with PDM. No significant minima were found with $\Theta$ less than 0.9 for any period larger than a day. (The residuals were also searched for periods supporting a doublemode pulsator interpretation without success.) Efforts to detect the beating of the Blazhko period against the primary period in both the original data and the residuals were also unsuccessful. However, this is not particularly surprising given the known irregularity of the Blazhko effect (see Smith 1995, pp. 107-109, for a discussion) and the limited sample available. Our data set spans 471 days, which covers only approximately four Blazhko cycles.

\section{CONCLUSIONS}

It is clear that FM Per is a multiperiodic RR Lyrae star. The variation in the magnitude of maximum light is caused by the Blazhko effect, and it is consistent with a Blazhko period of approximately 122 days. Hopefully, as the number of known RR Lyrae stars exhibiting the Blazhko effect increases, a correlation may be identified between the Blazhko effect and some other stellar parameter which could shed light on the physical mechanism causing the effect.

The instrumentation used for the observations described in this paper was funded by the National Science Foundation (grant AST 85-04072). Support for publication expenses was provided by the American Astronomical Society through its small grants program. This research made use of the SIMBAD database operated at CDS, Strasbourg, France.

\section{REFERENCES}

Blazhko, S. 1907, Astron. Nachr., 175, 325

Breger, M., Jaschek, C., \& Dubois, P. 1990, Inf. Bull. Variable Stars 3422

Clement, C. M., Kinman, T. D., \& Suntzeff, N. B. 1991, ApJ, 372, 273

Cousins, A. W. J. 1976, MmRAS, 81, 25

Cox, A. N. 1987, in IAU Colloq. 95, Second Conference on Faint Blue Stars, ed. A. G. D. Philip, D. S. Hayes, \& J. W. Liebert (Schenectady: Davis), 161

Garcia-Melendo E., \& Clement, C. M. 1997, AJ, 114, 1190

Jerzykiewicz, M., \& Wenzel, W. 1977, Acta Astron., 27, 35

Kholopov, P. N. 1985, General Catalogue of Variable Stars (4th ed.; Moscow: Nauka)

. 1987, General Catalogue of Variable Stars, Vol. 3 (4th ed.; Moscow: Nauka)
Landolt, A. U. 1983, AJ, 88, 439

Moskalik, P. 2000, in ASP Conf. Ser. 203, The Impact of Large-Scale Surveys on Pulsating Star Research, ed. L. Szabados \& D. W. Kurtz (San Francisco: ASP), 315

Nemec, J. M. 1985, AJ, 90, 204

Schmidt, E. G. 1991, AJ, 102, 1766

Schmidt, E. G., Chab, J. R., \& Reiswig, D. E. 1995, AJ, 109, 1239

Schmidt, E. G., \& Lee, K. M. 2000, PASP, 112, 1262

Smith, H. A. 1995, RR Lyrae Stars (Cambridge: Cambridge Univ. Press)

Stellingwerf, R. F. 1978, ApJ, 224, 953

Szeidl, B. 1988, in Multimode Stellar Pulsations, ed. G. Kovacs, L. Szabodos, \& B. Szeidl (Budapest: Konkoly Obs.), 45 